\title{
A construção de si como investigador: reflexões sobre os processos de formação pós-graduada ${ }^{1}$
}

\section{The construction of the self as a researcher: reflections on the process of post-graduate training}

\author{
Amelia Lopes* \\ Isabel Menezes*
}

\begin{abstract}
RESUMO
Este artigo discute os processos envolvidos na formação pós-graduada e o seu papel na construção de um sentido de si como investigador. Tendo como base dados recolhidos junto de estudantes de pós-graduação da Universidade do Porto e a experiência das autoras na direção de programas de pós-graduação em Ciências da educação, analisam-se algumas experiências relevantes do ponto de vista de uma aprendizagem situada da investigação. Atende-se especialmente as formas e dispositivos que envolvem professores, supervisores, colegas e outros investigadores e que podem contribuir para o desenvolvimento progressivo do que-fazer da investigação.
\end{abstract}

Palavras-chave: Pós-graduação. Mestrado. Doutorado. Investigação. Comunidade de prática de investigação.

1 Este trabalho é apoiado por fundos nacionais, através da Fundação para a Ciência e a Tecnologia, IP (FCT) e pelo Fundo Social Europeu (FSE), através do Programa Operacional do Capital Humano (POCH) de Portugal 2020, no âmbito do Programa de Doutoramento FCT em Ciências da Educação da Universidade do Porto; é ainda apoiado por fundos nacionais através da FCT, no âmbito do programa estratégico do Centro de Investigação e Intervenção Educativas (CIIE) com a ref. ${ }^{a}$ UID/CED/00167/2013. As autoras agradecem também à Doutora Carina Coelho a sua colaboração na recolha de alguns dos dados sobre o doutoramento.

* Universidade do Porto. Centro de Investigação e de Intervenção Educativas. Faculdade de Psicologia e de Ciências da Educação. Porto, Portugal. E-mail: amelia@fpce.up.pt. https://orcid. org/0000-0002-5589-5265. E-mail: imenezes@fpce.up.pt. https://orcid.org/0000-0001-9063-3773. 


\begin{abstract}
This paper discusses dimensions of post-graduated education and their role in the construction of a sense of self as a researcher, based on data collected with post-graduation students from the University of Porto and the experience of both authors as directors of post-graduation courses in Educational Sciences. We particularly focus on relevant experiences in for a situated learning of research, including the institution of research communities of practice that include professors, supervisors, researchers and post-graduation students and that can contribute to the progressive development of how-to-do research.
\end{abstract}

Keywords: Post-graduation. Master. PhD. Research. Research communities of practice.

Nas últimas décadas, na Europa como em outras regiões do mundo, registaram-se transformações intensas nos estudos pós-graduados. Essas transformações incluíram um acesso mais alargado a programas de mestrado e de doutoramento, um novo desenho de programas e cursos de pós-graduação, uma maior ênfase em competências transversais e nas qualificações direcionadas para o mercado de trabalho, a internacionalização e a mobilidade, e um apelo a projetos de investigação fundamentados quer nas profissionalidades quer nas práticas (KEHM, 2006; MALFROY, 2005; PARK, 2005; PEARSON; EVANS; MACAULEY, 2008; WALKER; GOLDE; JONES; BUESCHEL; HUTCHINGS, 2008). O caso português é particularmente interessante dado o aumento extraordinário da conclusão de mestrados e de doutoramentos (OECD, 2006; GOMES, 2010; SANTOS; HORTA; HEITOR, 2016), fator a que não é alheia a inclusão no espaço europeu de educação superior de que é sintoma maior o chamado "processo de Bolonha" (EUA-CDE, 2016; WELLINGTON, 2013), conforme Figura 1.

No que concerne ao doutoramento, entre 1990 e 2009, o número de novos doutorados, por ano, aumentou cinco vezes; em cada década, o número de doutoramentos mais do que tem duplicado e, entre 2010-2013, já tinha atingido mais de metade dos doutoramentos da década anterior, particularmente nas áreas das ciências exatas e naturais, ciências sociais e humanidades, ciências da engenharia e ciências médicas. No caso dos mestrados, a curva de crescimento que se verificou na década anterior à implementação da reforma de Bolonha, a partir de 2006/07, teve um crescimento exponencial por duas ordens de fatores: em primeiro lugar, alguns dos cursos de licenciatura pré-Bolonha com uma duração mais longa (cinco ou seis anos) foram reconfigurados como mestrados em áreas em que existia regulamentação europeia que o exigisse (e.g., Medicina, 
FIGURA 1 - EVOLUÇÃO DO NÚMERO DE DOUTORAMENTOS E MESTRADOS NOS ÚLTIMOS 20 ANOS

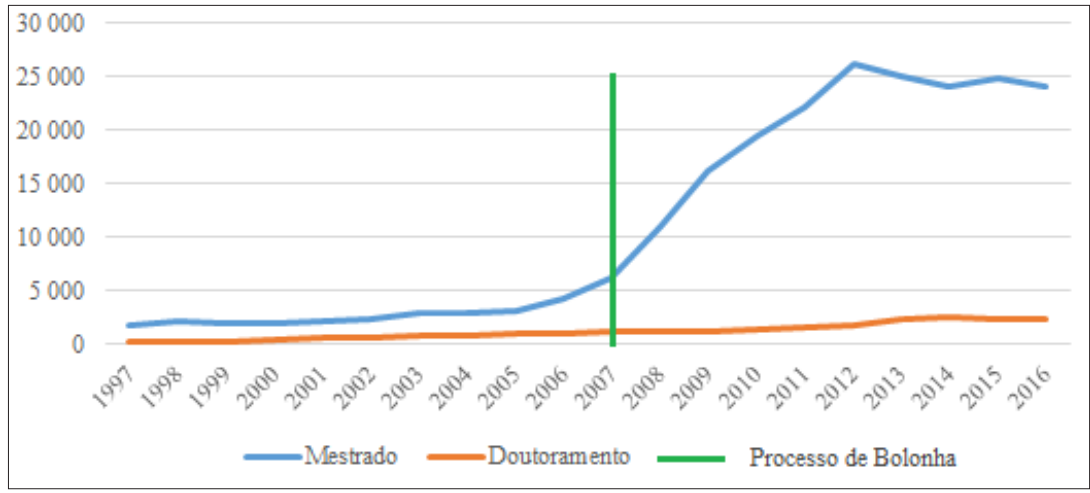

FONTE: Pordata (2018).

Engenharia, Arquitetura, Psicologia); em segundo lugar, a redução da duração da maioria das licenciaturas pré-Bolonha (de quatro para três anos) teve como consequência uma desvalorização do grau, tornando-se relativamente generalizada a ideia de que o mestrado era agora condição para o exercício profissional em qualquer área, junto de empregadores e de estudantes (SIN; TAVARES; AMARAL, 2016). Em geral, e mesmo que a percentagem de pós-graduados seja ainda baixa entre a população, existe uma crescente consciência da relevância do mestrado e do doutoramento, não só nos contextos académicos, mas também nos contextos profissionais (KOWALCZUK-WALĘDZIAK; LOPES; MENEZES; TORMENTA, 2017), situação que é similar à de outros países (KEMP; NEWNHAM; CHAPMAN, 2011). No caso específico dos professores, Portugal é o país da Europa em que o corpo docente apresenta qualificações mais elevadas - 12,4 \%, pois têm qualificação de nível 6 da International Standard Classification of Education (ISCED), correspondente à formação superior pós-graduada, no que é seguido pela República Checa com 4,5\% de professores com essa qualificação (CNE, 2016).

Ora, o processo de Bolonha veio reforçar essa articulação entre os saberes académicos e as profissionalidades, mesmo que se admita que esta não é uma questão propriamente nova. Por exemplo: no campo das Ciências da Educação - que será o contexto disciplinar do trabalho que aqui apresentamos - sempre foi reconhecida a importância de trabalhar na fronteira "entre a produção científica e a produção das práticas" (CORREIA, 2001, p. 33). No entanto, os descritores 
do Quadro Europeu de Qualificações ${ }^{2}$ enfatizam de forma especial a investigação como elemento distintivo da formação pós-graduada, quer ao nível dos conhecimentos, das aptidões ou das atitudes. Tanto ao nível do mestrado como do doutoramento, a investigação emerge como basilar a "um conhecimento altamente especializado" (no mestrado) ou a "um conhecimento de ponta na vanguarda de uma área de estudo ou de trabalho" (no doutoramento), mas simultaneamente como uma ferramenta estratégica para "resolver problemas complexos e imprevisíveis", sustentando a "inovação" e a produção de "novos conhecimentos e procedimentos" (mestrado) ou mesmo para lidar com "problemas críticos na área da investigação e/ou da inovação ou para o alargamento e a redefinição dos conhecimentos ou das práticas profissionais existentes" (doutoramento). A investigação é, assim e reconhecidamente, uma componente central da formação pós-graduada; saber se e como essa experiência de formação pode favorecer a construção de um sentido de si como investigador é o objetivo deste artigo, que parte da nossa experiência na direção de cursos de mestrado e de doutoramento na Universidade do Porto.

\section{Para uma pedagogia socioconstrutivista da investigação na universidade}

O lugar da investigação na universidade e a sua relação com o ensino têm sido objeto de intensa controvérsia, seja porque a dupla natureza enquanto professor e investigador tem raízes profundas na identidade dos docentes universitários e dos seus estudantes (BREW, 2003; ELTON, 2001; FLOUD, 2006; HENKEL, 2004), mas também porque se tem questionado a existência de uma relação entre as duas dimensões (e.g., HATTIE; MARSH, 1996) ou porque se advoga a supremacia do ensino (e.g., BERMAN BROWN, 2005; DEEM; LUCAS, 2006; KOGAN, 2004). Nos últimos anos, essa controvérsia está inevitavelmente relacionada com as políticas de "diminuição de recursos nos sistemas de ensino superior" (DEEM; LUCAS, 2006, p. 7), ao mesmo tempo que tem sido questionada a tradição europeia de que "a educação universitária é sinónima da investigação” (FLOUD, 2006, p. 11).

Os defensores de uma relação intrínseca entre investigação e ensino, salientam os efeitos positivos junto dos estudantes - tanto tangíveis, ao nível

2 http://www.anqep.gov.pt/aaaDefault.aspx $? \mathrm{f}=1 \& \mathrm{js}=0 \&$ codigono $=667166726679$ AAAA AAAAAAAA. 
dos conhecimentos e competências, como intangíveis, em termos de atitudes de questionamento e análise crítica -, advogando uma conceção do conhecimento como resultado de um processo de constante construção, com o contributo dos próprios professores (BREW, 1999, 2006; ELTON, 2001; HENKEL, 2004). Outros estudos têm avaliado o impacto do envolvimento em experiências de investigação (e.g., DEEM; LUCAS, 2006; ZAMORSKI, 2002), comprovando efeitos na aquisição de conhecimentos atualizados no domínio, na compreensão da "relação complexa e provisória entre investigação e conhecimento" (ZAMORSKI, 2002, p. 422), na promoção de competências de investigação e de conhecimento de métodos de investigação. Deem \& Lucas (2006, p. 13) reportam ainda avaliações positivas dos estudantes decorrentes da "experiência de ser ensinado por investigadores que partilham experiências e problemas, e de estudar num ambiente de intensa investigação". Henkel (2004) constata que os estudantes ao nível do mestrado tendem a estar mais intrinsecamente motivados para a investigação, embora salientando a importância da articulação entre a investigação e a prática profissional; uma exigência similar de utilidade e aplicabilidade é reportada por estudantes de pós-graduação no estudo de Lindsay, Breen e Jenkins (2002) - o que está em linha com a reconfiguração assumida pelo quadro europeu de qualificações ao qual nos referimos.

Os efeitos intangíveis podem tornar-se ainda mais salientes numa sociedade de conhecimento (HENKEL, 2004), na medida em que essas experiências propiciem a promoção de formas mais elaboradas de relação com o conhecimento e a "verdade científica", permitindo lidar com a incerteza e a complexidade (BREW, 1999; PERRY, 1970; BAXTER-MAGOLDA, 2004). O ensino pautado na investigação promove tanto o domínio de conhecimentos e competências especializados, quanto a partilha de esquemas conceptuais sobre o que significa fazer investigação num domínio; mas Brew e Boud (1995) salientam uma outra vantagem: a de aproximar a experiência dos professores e dos estudantes enquanto pessoas que aprendem, ou seja, enquanto aprendentes (BREW, 2006).

A relevância dessa articulação entre ensino e investigação ganha particular consistência se atendemos às perspetivas socioconstrutivistas e situacionais da aprendizagem (BRANSFORD; BROWN; COCKING, 2000; BROWN; COLLINS; DUGUID, 1989; GREENO; COLLINS; RESNICK, 1996; LAVE, 1988; PALINCSAR, 1998; RESNICK, 1988; ROGOFF, 1998; ROGOFF et al., 2003) que postulam a aprendizagem como indissociável da atividade, contexto e cultura em que decorre. Isso implica reconhecer que o conhecimento está "distribuído no mundo entre os indivíduos, ferramentas, artefactos, e livros que usam, e as comunidades de prática em que participam" (GREENO; COLLINS; RESNICK, 1996, p. 20) e que, para além de um conhecimento individual, existe também um conhecimento grupal e coletivo - podendo as práticas de um grupo 
facilitar ou inibir as atividades e a participação dos indivíduos, promovendo ou não o conhecimento.

A principal implicação para a formação em investigação é que seja dada aos estudantes oportunidade para uma "participação periférica legitima, o que envolve o acesso às práticas que é suposto aprenderem e a uma participação genuina nas atividades e preocupações do grupo" (GREENO; COLLINS; RESNICK, 1996, p. 23, grifo nosso), concebido assim como uma comunidade de aprendentes. Este é um processo gradual, em que os estudantes têm oportunidade de "observar e praticar atividades" que vão propiciar o desenvolvimento de competências e uma participação cada vez mais central - por meio de processos de modelagem, tutoria e esbatimento. Assim, os estudantes "participam ativamente na formulação e avaliação de questões e problemas, na construção e avaliação de hipóteses, evidências, argumentos e conclusões" (GREENO; COLLINS; RESNICK, 1996, p. 24). Hedegaard (1998, p. 121) concebe esse processo como "uma espiral de resolução de problemas" em que o professor começa por "iniciar os estudantes num domínio de conhecimento. Por intermédio do processo de aprendizagem, os estudantes" ganham protagonismo e "encontram os seus próprios problemas", isto é, envolvem-se na resolução de "problemas «situados» que são significativos em relação com o seu estádio desenvolvimental e situação... [supondo] formas de cooperação entre mestre e aprendiz e entre aprendizes" (HEDEGAARD, 1998, p. 120).

Três aspetos são essenciais à conceção da aprendizagem nessas perspetivas: a aprendizagem é vista como um processo de construção de conhecimento que depende de processos ativos de interpretação do mundo; a aprendizagem depende do conhecimento anterior que, quanto mais rico e elaborado, mais garante a qualidade dos processos de construção de novos conhecimentos; "os conhecimentos e competências não são independentes dos contextos físicos e sociais em que são usados" e dependem de uma "prática contextualizada" (RESNICK, 1988, p. 3).

Esses princípios são estruturantes para o desenho da formação em investigação nos cursos de pós-graduação em Ciências da Educação na Universidade do Porto. Desde logo, a importância atribuída a abordagens práticas que permitem 'pôr as mãos na massa' com graus crescentes de autonomia e complexidade, começando por experiências mais pontuais e supervisionadas (e.g., construir um guião de entrevista, de forma colaborativa, em situação de aula; realizar uma entrevista em conjunto com colegas), até a recolha de dados da sua própria investigação. Depois a ênfase na inclusão e articulação com o centro de investigação, em que as/os estudantes se inserem como investigadores juniores e são convidados a participar nas atividades de natureza científica (e.g., colóquios, seminários de apresentação e discussão de resultados de projetos). De 
particular relevância é a ênfase na integração de estudantes de pós-graduação em comunidades de prática de investigação que congregam orientadores e orientandos em diferentes momentos do seu percurso de mestrado e/ou de doutoramento, na partilha das suas experiências do que-fazer investigativo: os processos, incluindo tanto as dificuldades quanto os ganhos, as descobertas, as reconfigurações ... Finalmente, o estímulo e a provisão de oportunidades para apresentar o seu trabalho em eventos científicos, seja por meio da organização de seminários específicos para estudantes de pós-graduação, seja pelo estímulo à participação em congressos organizados por outras entidades.

\section{$O$ desenho da pesquisa: construir-se como investigador}

Como já referido, a formação pós-graduada dirige-se hoje especialmente a profissionais, quer já empregados quer em procura de emprego, uma vez que ela se tornou uma componente da formação profissional nas sociedades do conhecimento. Efetivamente, no contexto da Faculdade de Psicologia e de Ciências da Educação da Universidade do Porto (FPCEUP), a maioria dos estudantes de doutoramento ou mestrado em Ciências da Educação tem já uma ocupação ou procura a formação para tornar mais provável uma futura ocupação, a qual poucas vezes se dirige ao mundo académico. Coloca-se assim necessariamente o problema da articulação entre a formação em investigação e a formação profissional e mais especificamente o da formação de uma identidade (profissional) investigadora, ou seja, da construção de si como investigador/a.

Apresentaremos os resultados de dois estudos realizados com o objetivo de melhor conhecer os processos subjacentes à construção dessa identidade de investigador/a num contexto específico, o da formação pós-graduada em Ciências da Educação na FPCEUP - com enfoque no Programa Doutoral em Ciências da Educação e no Mestrado em Ciências da Educação.

O primeiro (Estudo 1) centra-se em antigos estudantes de doutoramento, muitos com mestrado realizado também na FPCEUP, e envolve uma combinação de entrevistas por telefone $(n=21)$ e de pequenas narrativas $(n=6)$ construídas para dar resposta às seguintes perguntas: "(1) Ao longo do seu percurso de formação pós-graduada (mestrado e/ou doutoramento) quais foram os momentos - o primeiro e os seguintes - em que se sentiu 'investigador/a'?"; “(2) Partilhe ainda alguns detalhes: Em que contexto(s) isso aconteceu? Estava sozinha/o ou com outras pessoas? O que estava a fazer?". 
O segundo (Estudo 2) incide na análise de grupos focais com professores do ensino primário ou médio, mestres ou doutores que se graduaram na FPCEUP nos cursos referidos; esses grupos focais tinham o intuito de aprofundar o conhecimento sobre o impacto da formação pós-graduada na atividade profissional e sua relação com componentes estruturais e dinâmicas da situação da formação.

Começaremos por descrever sucintamente a organização e o rationale da formação nos cursos em foco, Programa Doutoral em Ciências da Educação (PDCE) e Mestrado em Ciências da Educação (MCED), para depois nos debruçarmos especialmente sobre os resultados desses dois estudos.

\section{O contexto da pesquisa: o rationale e a estruturação da formação}

A criação de cursos de doutoramento é, em Portugal, um fenómeno relativamente recente. Com efeito, a forma clássica de realização de doutoramento - sendo que o primeiro doutoramento em Ciências da Educação é o de António Simões na Universidade de Coimbra em 1979 (RIBEIRO; MENEZES, 2017) -, envolvia a concretização de um projeto de investigação pessoal sob a orientação de um/a professor/a universitário/a. O processo era fortemente individualizado, não implicando a frequência de aulas ou cursos. A criação de um Programa de Doutoramento em Ciências da Educação, na Universidade do Porto, data de 1999, com a coordenação de Stephen R. Stoer, Luíza Cortesão e Helena C. Araújo, a que se seguiram outras edições coordenadas por outros professores; este programa envolvia já seminários coletivos com estudantes de doutoramento e ensaiava dispositivos de orientação coletiva, de forma a permitir a professores/as mais jovens iniciarem, de modo apropriado, processos de orientação de doutoramento.

Em 2006/2007, com o processo de Bolonha, foi formalmente criado um curso de doutoramento, com a duração de três anos, incluindo componentes curriculares ligadas (i) à epistemologia e metodologias de investigação, (ii) ao aprofundamento teórico no campo das ciências sociais e da educação e (iii) à escrita científica, mas também prevendo a apresentação de trabalhos em congressos; o curso tem um enfoque generalista, sendo que o espaço de especialização acontece nos seminários de investigação e nos seminários temáticos. A componente letiva é mais intensa no primeiro ano do curso, diminuindo gradualmente ao longo do segundo ano e sendo inexistente no terceiro ano, em que a/o doutorando está focado/a na escrita de tese; sendo verdade que a duração do curso é de três anos, a grande maioria de estudantes que frequenta o curso a tempo 
integral realiza o doutoramento em quatro anos. A conclusão do doutoramento exige, para além de uma tese original, que a/o doutorando tenha publicado, sobre a sua investigação, pelo menos um artigo científico em revista indexada, com peritagem, e tenha apresentado pelo menos três comunicações em reuniões científicas inter/nacionais. Todo esse processo envolve uma articulação muito próxima com a unidade de investigação em Ciências da Educação da FPCEUP, o Centro de Investigação e Intervenção Educativas (CIIE), reconhecendo os estudantes como investigadores juniores e promovendo o seu envolvimento ativo em atividades e projetos em curso.

$\mathrm{Na}$ Universidade do Porto, o Mestrado em Ciências da Educação existe desde 1992 e sofreu adaptações associadas ao processo de Bolonha em 2007. Nessa adaptação, o $1^{\circ}$ ano do curso foi semestralizado e passou a ter uma componente geral e outra específica, patente sobretudo no primeiro dos seus dois anos de duração. O primeiro ano coloca a ênfase em conteúdos de natureza teórica e de ênfase educacional (pelo menos 30 créditos), epistemológica e metodológica (de 18 a 26 créditos). A ênfase educacional é geral - quando aborda, por exemplo, a temática da cidadania e da diversidade - mas também específica, nas suas especializações. As especializações são constituídas por três disciplinas num total de 24 créditos (no $1^{\circ}$ ano) associadas a áreas de investigação do CIIE. Dessa forma têm existido especializações nos domínios de educação e exclusão, educação de adultos, intervenção comunitária, educação e género, formação de professores, educação prioritária, supervisão e liderança, entre outros. No segundo ano, a aquisição do grau pode ser realizada através de estágio - geralmente frequentado por jovens estudantes que procuram um primeiro emprego - ou através de uma dissertação, um trabalho de investigação com dimensões de originalidade ainda que de extensão limitada - sendo esta possibilidade frequentada sobretudo por profissionais. Num e noutro caso o processo de aprendizagem envolve 52 créditos.

A relação mais sistemática da formação pós-graduada com a investigação passou a ser considerada uma preparação crucial para a economia do conhecimento (WOOD, 2009) - embora, o lugar da investigação como componente pedagógica da formação seja assunto de debate na comunidade académica, dando origem a posições nem sempre convergentes. A principal questão concerne exatamente o grau em que a investigação é obstáculo ou contributo incontornável para a formação profissional superior. Trata-se de uma discussão complexa, mas claramente dependente das conceções de investigação que a informam. Nesse debate, e indo ao encontro da conceção socioconstrutivista sobre o lugar da investigação na formação superior acima apresentada, Brew $(2001,2010)$ e Wood $(2009)$ defendem uma conceção comunicativa de investigação, em que os estudantes são vistos como participantes da pesquisa, sendo, 
com os seus professores, membros da mesma "comunidade de aprendizagem" (ver LOCKE, 2012; WOOD, 2009). Adotando essa perspetiva "pedagógica" da investigação Healey e Jenkins (2006) apresentam quatro tipos de relação que os estudantes podem ter com a investigação: dirigida pela investigação (research-led), orientada pela investigação (research-oriented), baseada na investigação (research-based) e formação em investigação (research-tutored), envolvendo escrita e discussão de artigos. Para Healey e Jenkins (2006), as experiências de aprendizagem mais efetivas envolvem as quatro modalidades, com ênfase nas duas últimas que contemplam a participação dos estudantes. Ora, o rationale da organização dos dois cursos segue essa perspetiva, com vários aspetos em comum, independentemente da sua intensidade variar em função do curso:

- importância atribuída a uma formação aprofundada em metodologias de investigação, teoria educacional geral e específica, com ênfase nas ciências da educação;

- ênfase na ligação ao CIIE, através do estímulo ao conhecimento dos projetos em curso e da participação regular nas suas atividades científicas;

- foco em temas da atualidade educacional e em trabalhos de campo;

- organização de aulas abertas com investigadores de renome nas áreas temática em apreço:

- contacto frequente e com investigadores, em conferências e workshops de ênfase teórica ou epistemológica;

- compromisso da comunidade docente alargada na orientação inicial dos estudantes, de forma a permitir um período de exploração livre de potenciais temáticas e orientadores;

- após definição dos orientadores, inserção dos estudantes em comunidades de prática de investigação que envolvem um/a ou mais orientadores/as e o conjunto de estudantes de mestrado e/ou doutoramento que orientam;

- discussão coletiva e subsequente definição de um conjunto de normas orientadoras da supervisão que explicitam as expectativas face aos papéis de orientador/a e orientado/a.

\section{Estudo 1: a investigação no centro da experiência de pós-graduação}

No âmbito da monitorização do PDCE, foram realizadas entrevistas telefónicas (n=21) a doutorados em Ciências da Educação entre 2013 e 2017; a 
amostra foi constituída de forma aleatória, correspondendo a $43 \%$ do universo do total de doutorados nesse período, e indo ao encontro da diversidade de perfis de doutorados (nacionalidade, bolsa, tempo integral/parcial). A maioria dos participantes são mulheres $(57 \%)$, de nacionalidade portuguesa $(76 \%)$ e com bolsa durante o doutoramento (52\%), sendo que apenas três pessoas $(14 \%)$ não tinham uma formação anterior (licenciatura e/ou mestrado) em Ciências da Educação. O objetivo deste estudo era conhecer as motivações para o ingresso no PDCE e a sua avaliação sobre o impacto do doutoramento em termos pessoas e/ou profissionais e os elementos considerados mais relevantes no processo.

A escolha do PDCE parece dever-se à qualidade dos/as potenciais orientadores/as, à reputação da FPCEUP no que concerne à investigação que aí se produz, mas também à conhecida abrangência dos seus temas de investigação e favorecimento de abordagens interdisciplinares - o que é congruente com algumas preocupações do desenho curricular do PDCE e da própria conceção de investigação em Ciências da Educação dominante no CIIE.

Quanto ao impacto, a dimensão de se tornar um "estudioso" ou "académico" (scholar) numa determinada área é particularmente saliente, bem como o domínio aprofundado de metodologias de investigação. O doutoramento parece ter contribuído para uma visão mais abrangente, complexa, aprofundada, mas também mais aberta, sobre as questões educacionais que foram objeto de estudo, gerando um sentido de maior autonomia, autoria e autoridade para produzir um discurso nessa área temática. Essa autonomia, autoria e autoridade parece também caracterizar a forma como a/os entrevistados/as veem a sua capacidade para tomar decisões metodológicas e conduzir novos estudos, incluindo em relação à melhoria das suas práticas profissionais. O doutoramento é ainda referido como uma oportunidade de viver, por dentro, a experiência da academia, participando das experiências da faculdade e do centro da investigação, de acompanhar e realizar investigação rigorosa e de observar de perto o funcionamento de um centro de investigação. Parece-nos de realçar o destaque atribuído pelos/as entrevistado/as ao desafio intelectual de realizar o doutoramento, mas também a processos de natureza relacional decorrentes da interação com colegas, professores e investigadores diversos.

A entrevista telefónica incluiu também um questionário centrado nas competências e capacidades adquiridas, com base no Inquérito Pedagógico da Universidade do Porto (IPUP; SERRA LEMOS; MENEZES; TEIXEIRA; QUEIRÓS, 2014), em que essas dimensões são sistematicamente exploradas; como se pode observar na Tabela 1, embora haja uma valoração muito positiva de todas as competências, a relação com a investigação emerge como especialmente saliente. 
TABELA 1 -CAPACIDADES E COMPETÊNCIAS DESENVOLVIDAS AO LONGO DO DOUTORAMENTO

\begin{tabular}{|c|c|c|c|c|c|}
\hline & $\mathrm{N}$ & Mínimo & Máximo & Média & $\begin{array}{l}\text { Desvio } \\
\text { padrão }\end{array}$ \\
\hline $\begin{array}{l}\text { Capacidade para realizar trabalho de investigação } \\
\text { original que contribua para o alargamento do } \\
\text { conhecimento nesta área }\end{array}$ & 21 & 4 & 5 & 4,67 & ,483 \\
\hline $\begin{array}{l}\text { Aprofundar e estruturar conhecimentos avançados } \\
\text { no domínio das Ciências da Educação }\end{array}$ & 21 & 3 & 5 & 4,57 &, 598 \\
\hline $\begin{array}{l}\text { Conceber, projetar, adaptar e realizar uma } \\
\text { investigação significativa, respeitando as exigências } \\
\text { impostas pelos padrões de qualidade, inovação e } \\
\text { integridade académicas }\end{array}$ & 21 & 4 & 5 & 4,57 &, 507 \\
\hline $\begin{array}{l}\text { Desenvolver investigação de forma independente } \\
\text { para resolver problemas do seu quotidiano }\end{array}$ & 21 & 3 & 5 & 4,33 & ,730 \\
\hline $\begin{array}{l}\text { Capacidade de considerar as implicações éticas, } \\
\text { sociais e políticas na concretização de projetos de } \\
\text { investigação/intervenção }\end{array}$ & 21 & 2 & 5 & 4,29 & ,902 \\
\hline Capacidade de escrita académica & 21 & 2 & 5 & 4,19 & ,750 \\
\hline $\begin{array}{l}\text { Conhecimentos e competências de métodos de } \\
\text { investigação diversificados e atualizados na área }\end{array}$ & 21 & 4 & 5 & 4,14 & ,359 \\
\hline $\begin{array}{l}\text { Produzir conhecimentos interdisciplinares } \\
\text { adequados à análise e intervenção de situações de } \\
\text { educação e formação marcadas pela complexidade }\end{array}$ & 21 & 3 & 5 & 4,14 & 655 \\
\hline $\begin{array}{l}\text { Capacidade de comunicar temas científicos com } \\
\text { públicos especialistas na área }\end{array}$ & 21 & 1 & 5 & 4,10 & ,944 \\
\hline $\begin{array}{l}\text { Oportunidades para o estabelecimento de contactos } \\
\text { com investigadores/grupos de investigação }\end{array}$ & 21 & 1 & 5 & 4,10 & 1,044 \\
\hline $\begin{array}{l}\text { Oportunidades para divulgação do seu trabalho } \\
\text { de investigação junto de comunidades científicas } \\
\text { nacionais e internacionais }\end{array}$ & 21 & 1 & 5 & 4,00 & 1,140 \\
\hline $\begin{array}{l}\text { Capacidade de comunicar temas científicos com } \\
\text { públicos não especialistas na área }\end{array}$ & 21 & 2 & 5 & 3,95 & ,865 \\
\hline
\end{tabular}

FONTE: autoras (2018).

Finalmente, quando inquiridos sobre os aspetos mais e menos positivos do curso de doutoramento, é novamente a dimensão de investigação que aparece mais saliente - seja, pela positiva, pela oportunidade de integrar as já referidas comunidades de prática de investigação, de envolvimento em projetos de investigação em curso no CIIE, de conhecer os projetos desenvolvidos pelos professores da FPCEUP, de contactar com investigadores diversos de diferentes instituições, de apresentar trabalhos em congressos ou de ter apoio para submeter artigos; pela negativa é referida a necessidade de um maior aprofundamento 
e articulação entre a investigação e as práticas profissionais, mas também um maior reforço do apoio à escrita e à disseminação dos resultados da investigação.

Pareceu-nos que faria sentido complementar esses dados com narrativas sobre o/s momento/s em que, ao longo do doutoramento, cinco antigos estudantes da FPCEUP se "sentiram como investigadores". É muito interessante constatar, nessas narrativas, a presença de elementos próprios ao trabalho de pesquisa propriamente dita - a "descoberta de fontes (históricas/documentais) inéditas" -, mas também ao seu impacto na comunidade académica - "citação do nosso trabalho em publicações, trabalhos académicos e outros". Ou seja, sentir-se investigador parece ser um processo marcado pela continuidade de experiências diversas, como "entrevistar uma figura pública, conduzir grupos de discussão, fazer apresentações em conferências, publicar artigos, etc." - embora, neste caso, a dimensão relacional pareça ser prevalecente: "o processo que descrevi [...] ocorreu, na sua grande parte, na ou a partir da faculdade e quase sempre num ambiente de trabalho coletivo/colaborativo; inclusive o processo de escrita."

Dois aspetos singulares que emergem nessas narrativas merecem a nossa atenção - sempre com a investigação no centro. Num dos casos, é especialmente desafiante a ligação do projeto a problemas sentidos no passado na primeira pessoa, como neste caso em que o contexto hospitalar é (re)visitado:

[...] o que me levava ali era outro motivo, as pessoas que esperava eram outras - não eram profissionais de Saúde, eram utentes - e o que pretendia saber não era sobre mim, mas sobre elas. Objetivar um novo desempenho fez com que, passados alguns meses, me sentisse investigadora.

Num outro caso, é a atenção dos média a uma apresentação num congresso que despoleta um novo reconhecimento do que é ser investigadora:

No fim-de-semana seguinte fui a X., de onde sou natural, e no café várias pessoas falaram comigo sobre a notícia do jornal. Para aquelas pessoas, que eu conheço desde sempre e com as quais já havia conversado inúmeras vezes sobre 'ser investigadora', aquela foi claramente a primeira vez que se conseguiram relacionar com aquilo que faço, a investigação. Quiseram saber mais, fizeram mais perguntas e falaram comigo sobre as suas experiências e relações com a política. Experienciei, portanto, o impacto que a divulgação alargada, possível através dos meios de comunicação, pode ter no reconhecimento social da investigação: pôr as pessoas a falar sobre o assunto e a querer saber mais - não só sobre a participação 
política, mas também sobre a investigação nas ciências da educação. De repente, testemunhei a tal transferência de conhecimento científico desde a Universidade do Porto até um café em X.

\section{Estudo 2: formação pós-graduada e formação do professor investigador}

A investigação tem sido apontada como competência inerente ao perfil profissional do professor. Surgem referências a essa competência em diversos documentos de política educativa que enformam a carreira docente (DECRETO-LEI N. ${ }^{\circ} 241 / 2001$ ) e na literatura científica a investigação é insistentemente referida como condição de fortalecimento do profissionalismo dos professores - que enfatiza a necessidade do reconhecimento da atividade docente como uma profissão e dos professores como profissionais. Assim entendido, o profissionalismo implica autonomia política e científica da profissão e a autoria dos professores no que concerne as orientações e atos profissionais (LOPES; DOTTA, 2015; COCHRAN-SMITH, 2005; REIS-JORGE, 2005; BUCHBERGER; CAMPOS; KALLÓS; STEPHENSON, 2000; TARDIF; LESSARD; GAUTHIER, 1999). Recentemente, diversos estudos (DARLING-HAMMOND, 2017; ELLIS; MCNICHOLL, 2015; LOPES; BOYD; ANDREW; PEREIRA, 2014) estabelecem uma ligação direta entre a formação dos professores em investigação e a qualidade dos sistemas educativos, incluindo os resultados dos alunos. Nesses estudos, o sentido dado à investigação enfatiza exatamente o processo, os procedimentos e a atitude de investigação (a aprendizagem e o uso rigoroso e adequado de métodos de recolha e análise de dados, assim como o domínio de todos os passos do processo de investigação) e não apenas a aplicação pedagógica de evidências de investigação (RASMUSSEN; BAYER, 2014; DRENNAN; CLARKE, 2009). Por outro, a investigação é vista como parte (e informante) do desenvolvimento profissional, individual e na sua relação com a inovação e efetividade da escola, nomeadamente envolvendo processos de investigação colaborativa (COOPER; COWIE, 2009).

Neste estudo 1, professores em exercício que realizaram o doutoramento ou o mestrado na FPCEUP foram inquiridos em quatro grupos de discussão focalizada, com um total de 16 professores dos diferentes níveis de ensino. O objetivo central era estudar os efeitos que a formação pós-graduada teria tido na sua atividade profissional individual e a nível da escola, sendo uma das questões de investigação, entre outras, a seguinte: como se relacionam as competências e as identidades de investigação com as práticas nucleares dos professores? 
Os professores participantes começaram por escrever individualmente sobre as motivações que os levaram a frequentar o mestrado/doutoramento, para depois serem envolvidos coletivamente na discussão sobre os temas: a atividade profissional depois do mestrado e do doutoramento; o processo de realização da dissertação de mestrado/da tese de doutoramento; o professor investigador, os saberes académicos e os saberes práticos.

Os discursos produzidos encarados como "formações de linguagem" (DEJOURS, 1995) foram sujeitos a uma análise qualitativa de conteúdo de tipo temático informada pela análise conversacional (GUMPERZ, 1989). Da análise, dedutiva e indutiva, emergiu um sistema categorial a que reconhecemos o caráter de ideal-tipo. Este ideal-tipo é constituído por cinco dimensões - motivações; aprendizagem da investigação; impacto da formação avançada; conceções de professor investigador; e conceções de conhecimento - e três níveis, correspondentes às perceções identificados nos professores com o grau de mestre, com o grau de mestre e no momento a realizar estudos de doutoramento, e com o grau de doutoramento.

De seguida, vamos descrever alguns desses resultados, focando os aspetos mais diretamente relacionados com a investigação.

Como em Kowalczuk-Walędziak, Lopes, Menezes e Tormenta (2017), os participantes consideram ser necessária uma forte motivação para iniciar estudos avançados, dada a falta de apoio e reconhecimento que experimentam no seu contexto escolar. Para os professores mestres a motivação principal consiste em fugir à rotina e promover o desenvolvimento profissional. Os restantes participantes colocam a ênfase nessa dimensão pessoal do desenvolvimento profissional. O impacto da formação avançada abrange três componentes: uma nova atitude e competência profissional; novas relações com os colegas; e assunção de novas tarefas e responsabilidades. Os mestres reportam um empoderamento pessoal gerador de uma nova atitude profissional que lhes permite confrontar melhor o quotidiano profissional; reportam também a existência de uma outra e maior capacidade para justificar opções e ações, de ler e pesquisar para tomar decisões, de pensar e de pensar com flexibilidade, e de ser crítico. Dizem também compreender melhor os colegas de trabalho, terem ficado sensíveis à necessidade de os envolver em novos projetos e ter aprendido a ver os conflitos como presença de diferentes pontos de vista.

Os doutores sentem-se também pessoalmente empoderados, com mais coragem para defender as suas ideias e projetos e com mais capacidade para legitimarem as suas opções e atividades, mas parecem também terem-se tornado menos tolerantes ao statu quo (“deu-me mais coragem para me zangar", uma participante). Por vezes assumem novas funções, mas quando isso não acontece, investem na continuação da sua formação avançada (pós-doutoramento) ou em projetos de investigação. 
No que diz respeito à aprendizagem da investigação, todos os participantes se referem positivamente ao conhecimento educacional e ao ambiente investigativo que encontraram na FPCEUP, os quais consideram terem sido os principais responsáveis pela mudança de atitude que os tornou mais capazes e seguros nos seus pontos de vista e ações nas escolas. O conhecimento educacional a que puderam aceder (nas aulas, nas conferências, nas equipas e desenvolvendo investigação) é visto como um tipo específico de conhecimento educacional a que não tinham tido acesso antes (na formação inicial ou contínua) - é um conhecimento global e consistente, dando lugar a novas compreensões do que se passa em educação. $\mathrm{O}$ ambiente de investigação que respiraram é feito das relações de proximidade entre professores e estudantes e com outros investigadores nacionais e internacionais

Para os mestres, no processo de aprender a investigar, a dissertação é uma referência incontornável. Falam do quanto sofreram e do papel central dos supervisores - sobretudo no início e no fim, dando apoio afetivo, calendarizando tarefas e ensinando a escrever (ver DRENNAN; CLARKE, 2009). Para eles, o melhor de todo o processo foram as leituras prévias, a recolha e a análise de dados e a discussão pública da dissertação; o mais difícil foi "juntar tudo e escrever".

Os doutores não referem o mesmo sofrimento que os mestres. Tendo a maioria dos participantes realizado já o mestrado, o processo de doutoramento surge menos problemático. Referem, entretanto, a importância do orientador em todo o processo e como puderam fazer escolhas próprias a esse propósito; mas fica claro que se tratou de um trabalho pessoal para o qual contaram com todo um contexto com as características já referidas.

No que diz respeito às conceções de professor investigador, os participantes indicam três níveis de relação dos professores com a investigação: ausência de qualquer tipo de atitude investigativa; abertura à mudança e à inovação (o nível em que estavam quando decidirem frequentar o mestrado); e confirmação e fortalecimento dessa atitude com o mestrado (incluindo agora o conhecimento e o uso das metodologias de investigação propriamente ditas). Os professores doutorados acrescentam um outro nível, requerendo parcerias sustentadas (um ambiente de investigação) entre a academia e as escolas.

Em síntese, este estudo indica que a formação em investigação na formação avançada permite aos professores reafirmarem uma atitude inovadora, agir e ensinar de acordo com as reais necessidades do ensino e das escolas; compreender melhor as escolas e os comportamentos; escrever de forma científica projetos e outros textos (nomeadamente candidaturas a financiamento); procurar, ler e discutir para tomar decisões; e recolher e analisar informação (usando técnicas e métodos de análise aprendidos). Adaptar decisões aos contextos concretos e às perspetivas dos próprios professores e organizar e monitorizar a melhoria 
da escolha (quando em funções de coordenação) são atividades que os participantes consideram requerer especialmente competências de investigação de nível avançado. O perfil do profissional que assim se desenha aproxima-se do profissionalismo ativista referido em Loxley e Seery (2012).

\section{Conclusão}

A formação pós-graduada que esteve em análise neste texto organiza-se intencionalmente a partir de um referencial de investigação que responde aos desafios simultaneamente da qualidade da formação académica e do lugar da investigação no mundo atual. Ela mantém por isso níveis de exigência elevados nas componentes inerentes ao trabalho de investigação hoje ou ontem, ao mesmo tempo que aposta de forma clara e esclarecida em formas colaborativas, participativas e comunicativas do trabalho de investigação. Os estudos apresentados dizem que em geral estes objetivos têm sido atingidos ao mesmo tempo que elucidam sobre o impacto pessoal e profissional da formação de alto nível em investigação e sobre dimensões a investir, por exemplo, a articulação entre a formação em investigação e a disseminação da ciência ou as práticas profissionais.

O estudo do impacto pessoal e profissional da investigação, ou seja, da formação de si como investigador/a (para o mundo académico ou para o mundo profissional) revela o quanto a prática investigativa e os ambientes de investigação são cruciais na emergência de uma identidade investigadora; a este propósito enfatiza-se a experiência da academia por dentro, a proximidade com a investigação "a sério" por meio da integração nas equipas do centro de investigação, o rigor e a profundidade no método, e a robustez do conhecimento. $\mathrm{O}$ conhecimento educacional centrado nas ciências sociais e numa perspetiva articulada e crítica, as relações e o ambiente de investigação, mas também a supervisão próxima, organizada e distribuída (individual, nas comunidades de prática de investigação, nos grupos de investigação) são dimensões do currículo valorizadas.

Os estudos apresentados revelam ainda uma visão da investigação que claramente contempla a comunicação com a sociedade, com ênfase no impacto nos média e na importância da disseminação em geral.

A emergência da identidade de investigador (de investigadora) encontra-se na construção de competências específicas de investigação (técnicas), mas também na formação de atitudes de autonomia, autoria e autorização, quer na construção do conhecimento em sentido estrito e associado a identidades 
académicas (mais patente no Estudo 1), quer na construção do conhecimento profissional associado a identidades profissionais (mais patente no Estudo 2). O desafio intelectual, o processo relacional e a descentração de si surgem como dimensões importantes da construção de si como investigador/a, a que corresponde, interessantemente, também, uma relação com o conhecimento simultaneamente mais profunda e complexa e mais aberta e flexível.

\section{REFERÊNCIAS}

BAXTER-MAGOLDA, M. B. Evolution of a constructivist conceptualization of epistemological reflection. Educational Psychologist, v. 39, n. 1, p. 31-42, 2004.

BERMAN BROWN, R. Why link personal research and teaching? Education + Training, v. 47, n. 6, p. 393-407, 2005.

BRANSFORD, J. D.; BROWN, A. L.; COCKING, R. R. How people learn. Brain, mind, experience, and school. Washington, D. C.: National Academy Press, 2000.

BREW, A.; BOUD, D. Teaching and research: Establishing the vital link with learning. Higher Education, n. 29, p. 261-273, 1995.

BREW, A. Research and teaching: Changing relationships in a changing context. Studies in Higher Education, v. 24, n. 3, p. 291-311, 1999.

BREW, A. Conceptions of Research: a phenomenographic study. Studies in Higher Education, v. 6, n. 3, p. 271-285, 2001.

BREW, A. Teaching and research: New relationships and their implications for inquirybased teaching and learning in higher education. Higher Education Research \& Development, v. 22, n. 1, p. 3-18, 2003.

BREW, A. Research and teaching: Beyond the divide. Houndmills: Palgrave Macmillan, 2006.

BREW, A. Imperatives and challenges in integrating teaching and research. Higher Education Research \& Development, v. 29, n. 2, p. 139-150, 2010.

BROWN, J. S.; COLLINS, A.; DUGUID, P. Situated cognitionand the culture of learning. Educational Researcher, v. 18, n. 1, p. 32-41, 1989.

BUCHBERGER, F.; CAMPOS, B. P.; KALLÓS, D.; STEPHENSON, J. Green paper on teacher education in Europe: High quality teacher education for high quality education and training. Umea: TNTEE, 2000. 
CNE. A condição docente: contributos para uma reflexão (relatório técnico). Lisboa: Conselho Nacional de Educação, 2016.

COCHRAN-SMITH, M. The new teacher education: For better or for worse? Educational Researcher, v. 34, n. 7, p. 3-17, 2005.

COOPER, B.; COWIE, B. Collaborative research for assessment for learning. Teaching and Teacher Education, n. 26, p. 979-986, 2009.

CORREIA, J. A. A construção científica do político em educação. Educação, Sociedade \& Culturas, n. 15, p. 19-43, 2001.

DARLING-HAMMOND, L. Teacher education around the world: What can we learn from international practice? European Journal of Teacher Education, 40(3), 291-309, 2017.

DEEM, R.; LUCAS, L. Learning about research: Exploring the learning and teaching/ research relationship amongst educational practitioners studying in higher education. Teaching in Higher Education, v. 11, n. 1, p. 1-18, 2006.

DEJOURS, C. Analyse psychodynamique des situations de travail et sociologie du langage. In: BOUTET, J. (Ed.). Paroles au travail. Paris: L'Harmattan, p. 181-224, 1995.

DRENNAN, J.; CLARKE, M. Coursework master's programmes: the student's experience of research and research supervision. Studies in Higher Education, v. 34, n. 5, p. 483-500, 2009.

ELLIS, V.; MCNICHOLL, J. Transforming Teacher Education: Reconfiguring the academic work. London: Bloomsbury, 2015.

ELTON, L. Research and teaching: Conditions for a positive link [1]. Teaching in Higher Education, v. 6, n. 1, p. 43-56, 2001.

EUA-CDE. Doctoral education - taking Salzburg forward. Implementation and new challenges, 2016. Retrieved from: <http://www.eua.be/Libraries/publications-homepage-list/Doctoral-Education_Taking-Salzburg-Forward.pdf?sfvrsn=18>, on May 15, 2017.

FLOUD, R. The Bologna process: Transforming European higher education. Change, p. 8-15, 2006.

GOMES, J. F. (Org.). A ciência em Portugal - Relatório. Lisboa: Assembleia da República, 2010.

GREENO, J. G.; COLLINS, A. M.; RESNICK, L. B. Cognition and learning. In: BERLINER, D. C.; CALFEE, R. C. (Eds.). Handbook of educational psychology. New York: Macmillan, 1996. p. 15-45.

GUMPERZ, J. Engager la conversation: introduction à la sociolinguistique interactionnelle. Paris: Minuit, 1989.

HATTIE, J.; MARSH, H. W. The relationship between research and teaching: A metaanalysis. Review of Educational Research, v. 66, n. 4, p. 507-542, 1996. 
HEALEY, M.; JENKINS, A. Strengthening the teaching-research linkage in undergraduate courses and programs. New Directions for Teaching and Learning, n. 107, p. 45-55, 2006.

HEDEGAARD, M. Situated learning and cognition: Theoretical learning and cognition. Mind, Culture and Activity, v. 5, n. 2, p. 114-126, 1998.

HENKEL, M. Teaching and research: The idea of a nexus. Higher Education Management and Policy, v. 16, n. 2, p. 19-30, 2004.

KEHM, B. M. Doctoral education in Europe and North America: a comparative analysis. In: ULRICH, Teichler (Ed.). The Formative Years of Scholars. Wenner Gren International Series, 2006.

KEMP, J.; NEWNHAM, P.; CHAPMAN, E. The biomedical doctorate in the contemporary university: education or training and why it matters. High Educ., n. 63, p. 631-644, 2011.

KOGAN, M. Teaching and research: Some framework issues. Higher Education Management and Policy, v. 16, n. 2, p. 9-18, 2004.

KOWALCZUK-WALĘDZIAK, M.; LOPES, A.; MENEZES, I.; TORMENTA, N. Teachers pursuing a doctoral degree: motivations and perceived impact. Educational Research, v. 59, n. 3, p. 335-352, 2017.

LAVE, J. Cognition in practice: Mind, mathematics, and culture in everyday life. Cambridge, UK: Cambridge University Press, 1988.

LINDSAY, R.; BREEN, R.; JENKINS, A. Academic research and teaching quality: The views of undergraduate and postgraduate students. Studies in Higher Education, v. 27, n. 3, p. 309-327, 2002.

LOCKE, W. The dislocation of teaching and research and the reconfiguring of academic work. London Review of Education, v. 10, n. 3, p. 361-374, 2012.

LOPES, A.; BOYD, P.; ANDREW, N.; PEREIRA, F. The research-teaching nexus in nurse and teacher education: Contributions of an ecological approach to academic identities in professional fields. Higher Education, 68(2), 167-183, 2014.

LOPES, A.; DOTTA, L. T. Para um novo profissionalismo docente: Novos mapas e figuras da formação. In: LOPES, A.; PEREIRA, F.; QUEIROZ FREITAS, M. de; RIBEIRO DE FREITAS, A. (Eds.). Trabalho docente, subjetividade e formação. Porto: Mais Leituras, 2015. p. 157-166.

LOXLEY, A.; AND SEERY, A. The role of the professional doctorate in Ireland from the student perspective. Studies in Higher Education, v. 37, n. 1, p. 3-17, 2012.

MALFROY, J. Doctoral supervision, workplace research and changing pedagogic practices. Higher Education Research \& Development, v. 24, n. 2, p. 165-178, 2005. 
OECD. Tertiary education in Portugal. Background report. 2006. Retrieved from $<$ http:// www.oecd.org/dataoecd/21/17/39710472.pdf>, on Avril 2, 2012.

PALINCSAR, A. S. Social constructivist perspectives on teaching and learning. Annual Review of Psychology, n. 49, p. 345-375, 1998.

PARK, C. New variant PhD: the changing nature of the doctorate in the UK. Journal of Higher Education Policy \& Management, v. 27, n. 2, p.189-207, 2005.

PEARSON, M.; EVANS, T.; MacAULEY, P. Growth and Diversity in Doctoral Education: Assessing the Australian Experience. Higher Education, v. 55, n. 3, p. 357-372, mar., 2008.

PERRY, W. G. Forms of intellectual and ethical development in the college years. New York: Holt, Rinehart and Winston, 1970.

RASMUSSEN, J.; BAYER, M. Comparative study of teaching content in teacher education programmes in Canada, Denmark, Finland and Singapore. Journal of Curriculum Studies, 46(6), 798-818, 2014.

REIS-JORGE, J. Developing teachers' knowledge and skills as researchers: a conceptual framework. Asia-Pacific Journal of Teacher Education, v. 33, n. 3, p. 303-319, 2005.

RESNICK, L. B. (Ed.). Knowing, learning, and instruction: Essays in honour of Robert Glaser. Hillsdale, NJ: Erlbaum, 1988.

RIBEIRO, N.; MENEZES, I. A investigação em Ciências da Educação em Portugal nos últimos 30 anos: Evoluções, tendências e tensões vistas a partir das teses de doutoramento. Estado da Educação 2016. Lisboa: CNE - Conselho Nacional de Educação, p. 234-252, 2017.

ROGOFF, B. Cognition as a collaborative process. In: DAMON, W.; KHUN, D.; SIEGLER, R. S. (Eds.). Handbook of Child Psychology, $5^{\text {th }}$ ed., v. 2, p. 679-743. New York: Wiley, 1998.

ROGOFF, B.; PARADISE, R.; ARAUZ, R. M.; CORREA-CHÁVEZ, M.; ANGELILLO, C. Firsthand leaning through intent participation. Annual Review of Psychology, v. 54, n. 1, p. 175-203, 2003.

SANTOS, J. M.; HORTA, H.; HEITOR, M. Too many PhDs? An invalid argument for countries developing their scientific and academic systems: The case of Portugal. Technological Forecasting \& Social Change, n. 113, p. 352-362, 2016.

SERRA LEMOS, M.; MENEZES, I.; TEIXEIRA, P. M.; QUEIRÓS, C. Construção e validação do Inquérito Pedagógico da Universidade do Porto. Porto: FPCEUP, 2014.

SIN, C.; TAVARES, O.; AMARAL, A. Student perceptions of the employability of the first degree in Portugal. Education+ Training, 58(9), 966-983, 2016.

WALKER, G.; GOLDE, C. M.; JONES, L.; BUESCHEL, A. C.; HUTCHINGS, P. The Formation of Scholars: Rethinking Doctoral Education for the Twenty First, 2008. 
TARDIF, M.; LESSARD, C.; GAUTHIER, C. Formação dos professores e contextos sociais: perspetivas internacionais. Porto: Rés-Editora, 1999.

WELLINGTON, J. Searching for "doctorateness". Studies in Higher Education, v. 38, n. 10 , p. 1490-1503, 2013.

WOOD, D. Challenges to strengthening the teaching and research nexus in the first-year undergraduate curriculum. The International Journal of Learning, v. 15, n. 12, p. 111-120, 2009.

ZAMORSKI, B. Research-led teaching and learning in higher education: A case. Teaching in Higher Education, v. 7, n. 4, p. 411-427, 2002.

Texto recebido em 17 de maio de 2018 . Texto aprovado em 17 de julho de 2018 . 